\title{
Severe tracheobronchial stenosis in granulomatosis with polyangiitis and type 2 respiratory failure
}

\author{
Gayatri Ekbote (1), Rajiva Gupta (D), Natasha Negalur $(\mathbb{D}$, Dhaval Tanna (1) \\ Medanta The Medicity, Rheumatology \& Clinical Immunology, Gurgaon, India
}

Tracheobronchial stenosis (TBS) is seen in around $16-23 \%$ of granulomatosis with polyangiitis (GPA) patients and can often be a severe functional impairment and life-threatening event. ${ }^{1}$

Tracheobronchial stenosis can be a sole manifestation or progression that may not correlate with other GPA disease activity features and antineutrophil cytoplasmic autoantibody (ANCA) titers. ${ }^{2,3}$ This condition needs a multidisciplinary approach owing to its complexity. Apart from immunosuppression, multiple other modalities have been tried in the therapeutics of TBS associated with GPA. Tracheobronchial stenting is one of these.

A 50-year-old female patient was evaluated at our center in February 2016 with history of hearing loss, inflammatory arthritis, and saddle nose for four years and new-onset worsening of hoarseness of voice, cough and breathlessness for three months. She was being treated as GPA based on clinical features and anti-myeloperoxidase (MPO) positivity with oral steroids and weekly methotrexate of $22.5 \mathrm{mg}$. Further evaluation showed normal complete blood count but raised erythrocyte sedimentation rate with normal urine examination, renal functions and liver functions. Chest X-ray did not show any infiltrates. Cardiac evaluation was normal. High-resolution computed tomography (HRCT) of the chest with neck cuts showed circumferential thickening of trachea (Figure 1).

Considering this as a disease activity, the patient was started on intravenous
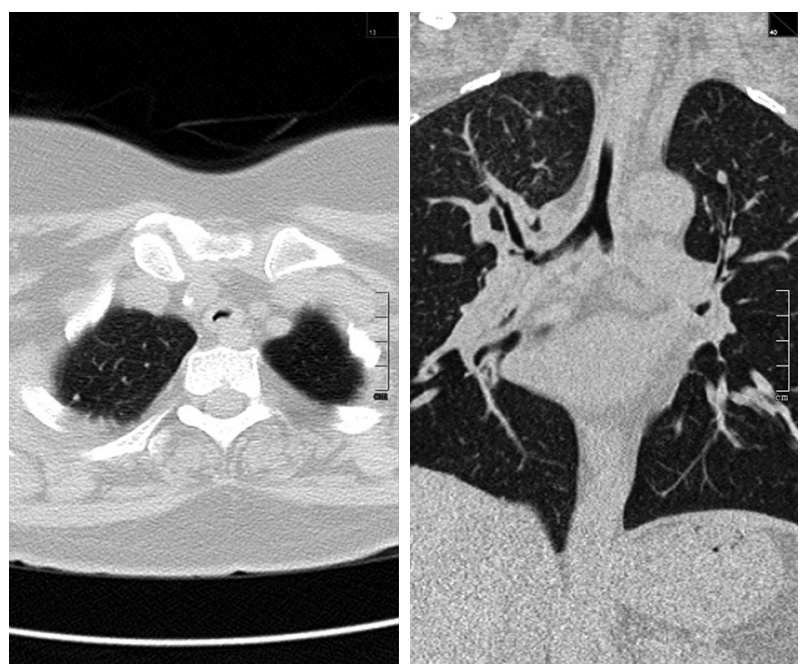

Figure 1. Changes shown more markedly in right bronchus.

Received: July 16, 2019 Accepted: January 02, 2020 Published online: June 24, 2021

Correspondence: Rajiva Gupta, MD. Medanta The Medicity, Rheumatology \& Clinical Immunology, 122006 Gurgaon, India. Tel: 09850285910 e-mail: guptarajiva@hotmail.com 

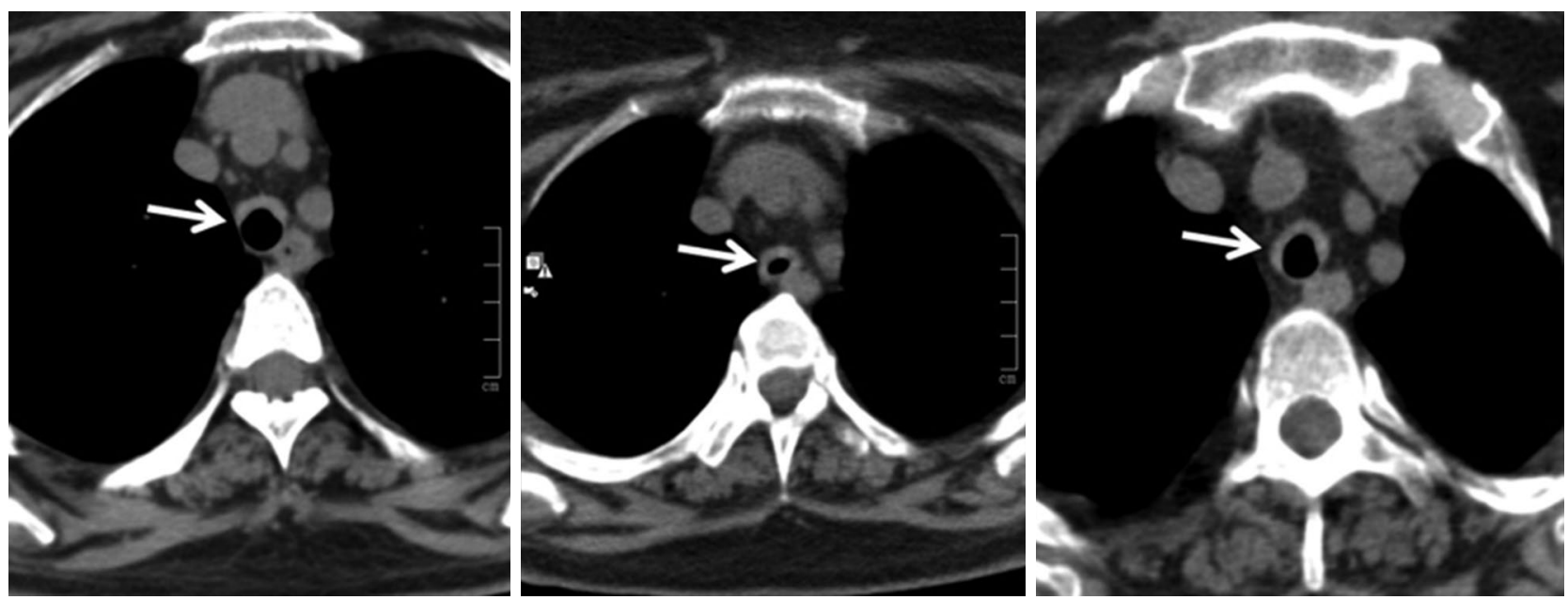

Figure 2. Improvement in wall thickening with rituximab.

cyclophosphamide $15 \mathrm{mg} / \mathrm{kg}$ monthly for six months and oral steroids $1 \mathrm{mg} / \mathrm{kg}$ with further tapering. She clinically became better and was on oral prednisolone $5 \mathrm{mg} /$ day and cotrimoxazole prophylaxis. She was planned for repeat HRCT of the chest and neck and maintenance treatment. However, the patient was lost to follow-up after six doses.

In January 2017, she presented with twoweek history of worsening of shortness of breath, wheeze and stridor. Further evaluation showed interval increase in circumferential thickening of trachea and bilateral main stem and lobar bronchi on HRCT of the chest and neck. However, repeat ANCA was negative for both proteinase 3 and MPO by line immunoassay and by indirect immunofluorescence for cytoplasmic and peri-nuclear ANCA. After initial treatment with broad-spectrum antibiotics and increased steroid dose, she had a marginal improvement. Considering this as a disease relapse, she was given rituximab $2 \mathrm{~g}$ two weeks apart. At subsequent follow-ups, the patient was doing well with just oral prednisolone $5 \mathrm{mg}$ daily. At fourth month follow-up, HRCT of the chest with neck cuts was repeated and it showed reduction in wall thickening as compared to the previous scans (Figure 2). This time, the patient was given early rituximab $500 \mathrm{mg}$ maintenance therapy and discharged on same dose of oral prednisolone and cotrimoxazole.

She was doing well for six months; however, she presented to emergency with history of cough, fever and wheeze for 15 days. On evaluation, she had type 2 respiratory failure and needed biphasic positive airway pressure support. Also, she was given high-dose steroids under antibiotics cover. HRCT of the chest showed worsening as expected. There was no improvement with the existing treatment even after three weeks. Patient continued having carbon dioxide retention with high respiratory rate and deterioration of

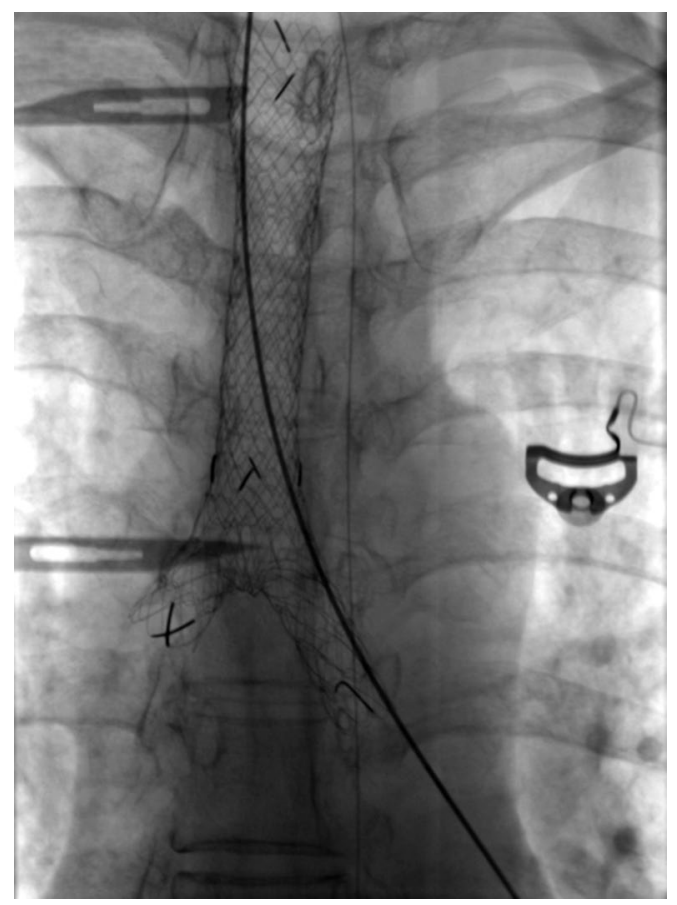

Figure 3. Ultraflex nitinol self-expandable stent. 


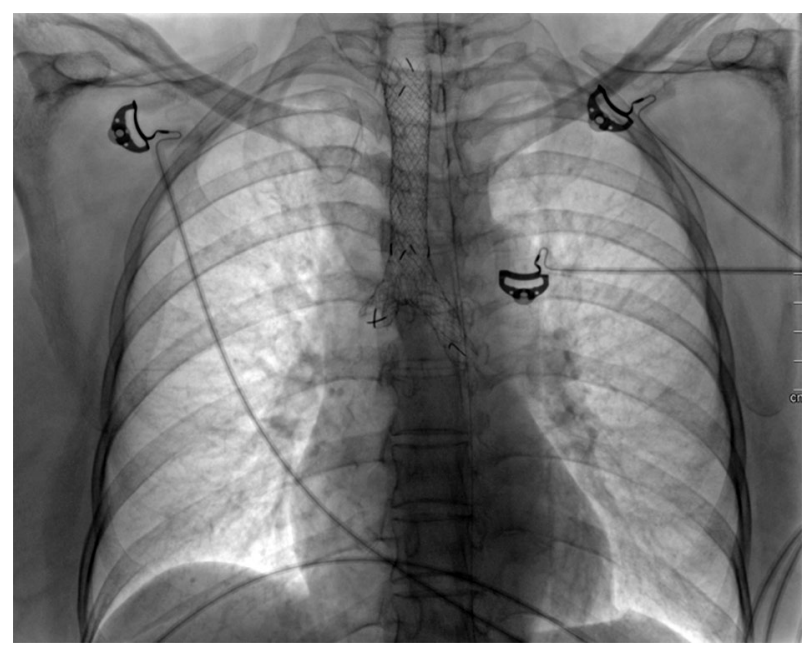

Figure 4. Post-procedure expansion of lung field.

sensorium despite noninvasive ventilation support. She also developed hospital-acquired infection. Discussion with the respiratory team and relatives ensued and decision for tracheal stenting was established (Figures 3 and 4).

Earlier stenting was avoided in view of long segment involvement. Post-procedure, she was put on ventilator and tracheostomized later in view of long-term ventilatory involvement. She was discharged after three weeks of hospital stay and later she suffered from post-procedure complications like tracheostomy site ulcer and its bleeding, subcutaneous emphysema and difficulty regaining activities of daily living. She was readmitted after a month with worsening of breathlessness and fever and was bound to have bilateral pneumonia. This time, she succumbed in view of sepsis and related acute kidney injury.

This case highlights the difficulties in managing TBS associated with GPA. Since GPA is notorious for higher relapse rates, presence of TBS makes it worse. TBS develops in watershed area, at the junction of two separate embryological different growth centers. Recurrent exposure of already inflamed respiratory epithelium to gastric contents during laryngopharyngeal reflux episodes leads to initial granulomatous inflammation which is followed by circumferential scarring and airway narrowing. Type 2 respiratory failure is not uncommon and can occur because of the involvement of segmental and small bronchi and bronchioles.

Endobronchial stenting also has a few complications including displacement of the stent and obstruction with secretions or granulation tissue. Rarely, the stent may perforate the airway wall, sometimes into the accompanying blood vessel. Most studies of endobronchial stenting demonstrate that it is an effective treatment modality for airway stenosis. The long-term efficacy of endobronchial stents is superior to laser ablation, debulking or dilatation therapy though the long-term patency of stents is uncertain. These patients often have ear, nose, and throat involvement and more commonly saddle nose deformity. TBS can progress in absence of any other systemic activity. Treatment modalities are limited and are as shown in Figure 5.,5

There are limited data on the usefulness of stenting in TBS associated with GPA. Two

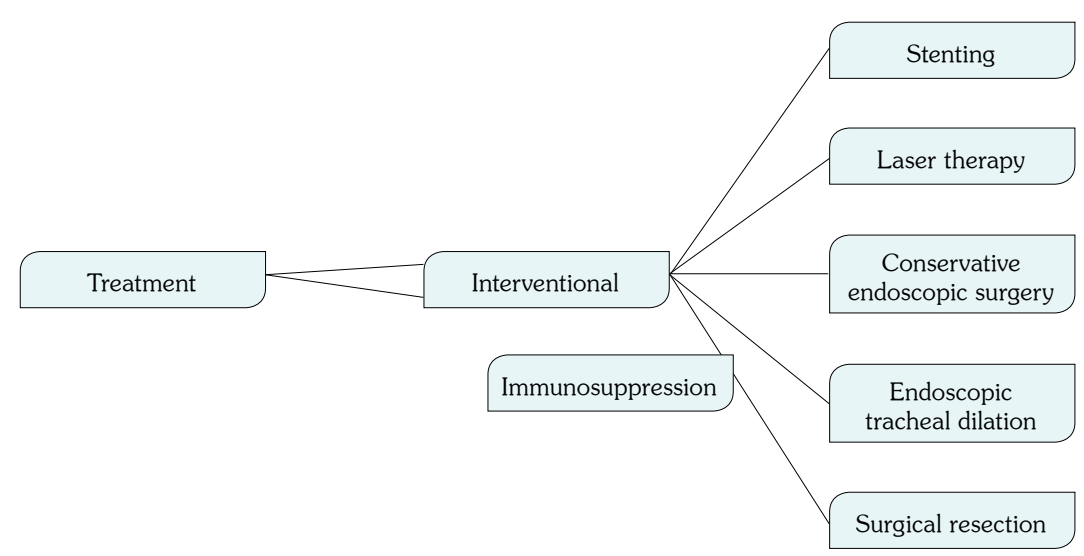

Figure 5. Current available treatment for tracheobronchial stenosis. 
recent studies in fact showed that the relapse rate was high as $62 \%$ with higher stent restenosis. ${ }^{6}$ Very few case reports have shown success with stenting. ${ }^{7,8}$

Considering that TBS can progress irrespective of systemic activity of GPA, it is difficult to assess response to immunosuppression with cyclophosphamide or rituximab etc. clinically.

\section{Acknowledgement}

We would like to thank the division of pulmonary medicine at Medanta The Medicity.

\section{Declaration of conflicting interests}

The authors declared no conflicts of interest with respect to the authorship and/or publication of this article.

\section{Funding}

The authors received no financial support for the research and/or authorship of this article.

\section{REFERENCES}

1. Martinez Del Pero M, Rasmussen N, Chaudhry A, Jani $\mathrm{P}$, Jayne D. Structured clinical assessment of the ear, nose and throat in patients with granulomatosis with polyangiitis (Wegener's). Eur Arch Otorhinolaryngol 2013;270:345-54.
2. Langford CA, Sneller MC, Hallahan CW, Hoffman GS, Kammerer WA, Talar-Williams C, et al. Clinical features and therapeutic management of subglottic stenosis in patients with Wegener's granulomatosis. Arthritis Rheum 1996;39:1754-60.

3. Stone JH. Wegener's Granulomatosis Etanercept Trial Research Group. Limited versus severe Wegener's granulomatosis: baseline data on patients in the Wegener's granulomatosis etanercept trial. Arthritis Rheum 2003;48:2299-309.

4. Fraga JC, Adil EA, Kacprowicz A, Skinner ML, Jennings $\mathrm{R}$, Lillehei $\mathrm{C}$, et al. The association between laryngeal cleft and tracheoesophageal fistula: myth or reality? Laryngoscope 2015;125:469-74.

5. Taylor SC, Clayburgh DR, Rosenbaum JT, Schindler JS. Clinical manifestations and treatment of idiopathic and Wegener granulomatosis-associated subglottic stenosis. JAMA Otolaryngol Head Neck Surg 2013;139:76-81.

6. Hernández-Rodríguez J, Hoffman GS, Koening CL. Surgical interventions and local therapy for Wegener's granulomatosis. Curr Opin Rheumatol 2010;22:29-36.

7. Girard C, Charles P, Terrier B, Bussonne G, Cohen $\mathrm{P}$, Pagnoux C, et al. Tracheobronchial Stenoses in Granulomatosis With Polyangiitis (Wegener's): A Report on 26 Cases. Medicine 2015;94:e1088.

8. Terrier B, Dechartres A, Girard C, Jouneau S, Kahn $\mathrm{JE}$, Dhote R, et al. Granulomatosis with polyangiitis: endoscopic management of tracheobronchial stenosis: results from a multicentre experience. Rheumatology (Oxford) 2015;54:1852-7. 